\title{
Energy-Efficient Design for Relay-Aided MIMO-OFDM Cognitive Radio Networks
}

\author{
B. Wu, J. Zuo, L. Zhao, C. Zou
}

\author{
Bin Wu*, Jiakuo Zuo, Li Zhao, Cairong Zou \\ School of Information Science and Engineering, \\ Southeast University, Nanjing, 210096, China \\ njwubin@seu.edu.cn, zuojiakuo85418@gmail.com \\ zhaoli@seu.edu.cn, cairong@seu.edu.cn \\ *Corresponding author:njwubin@seu.edu.cn
}

\begin{abstract}
With the explosive growth of high-rate multimedia services and promptly boomed energy consumption in wireless networks, energy-efficient design is become more and more important. In this paper, we investigate energy-efficient design for relay-aided multiple-input multiple-output-orthogonal frequency division multiplexing (MIMO-OFDM) cognitive radio networks. We formulate an energy-efficient power allocation problem, which takes a form of nonlinear fractional programming. To solve the problem, we first make a joint concave approximation to the original problem which facilitates the optimal algorithm development. Then, we derive an equivalent parametric optimization problem of the approximated problem. Finally, an iteration energy-efficient power allocation algorithm is presented. Numerical results reveal that the proposed algorithm can improve energy efficiency over traditional capacity maximization method.
\end{abstract}

Keywords: cognitive radio, power allocation, MIMO, relay, fractional programming.

\section{Introduction}

Cognitive radio (CR) and multiple-input multiple-output-orthogonal frequency division multiplexing (MIMO-OFDM) communications have been considered as a promising scheme to solve the spectrum scarcity problem and improve the quality of wireless communications [1]. Recently, to reduce the multi-path fading and improve the channel capacity, cooperative relaying technique is considered as a potent means to be adopted in the CR networks. Thus, the researches of relay aided MIMO CR networks are being received a growing attention in recent years $[2,3]$.

In [4], the relay selection and beamforming problem for the non-regenerative MIMO cognitive multi-relay network was considered and an optimal scheme was proposed via maximizing the capacity of the SUs by selecting the best cognitive MIMO relay. [5] studied a new paradigm for $\mathrm{CR}$ networks, which allowed the secondary users (SUs) to cooperatively relay the traffic for the primary users (PUs) while simultaneously transmitting their own traffic, and proposed a novel MIMO cooperative cognitive radio networks framework. [6] considered the power allocation problem for MIMO two-way CR sytem under a specturm sharing scenario, and presented an analytical expression of the optimal power allocation to each antenna of the treminals. [7] studied the power and channel allocation, and relay assignment for MIMO-OFDM based cooperative CR networks and proposed an optimal complexity algorithm and a sub optimal low complexity algorithm. [8] investigated subcarrier pairing and power allocation for MIMO-OFDM relay-aided CR networks and used environmental learning algorithm to mitigate the interference of the PUs. In the previous works, most researches intend to improve the throughput of relay aided CR systems. However, the energy efficiency (EE) has been considered more and more important in future wireless communication networks. The wireless devices and equipments consume about $9 \%$ of the total energy of information technology, the communication and information technology already contributes to about $2 \%$ of the global carbon dioxide emissions $[9,10]$. Therefore green 
communication, which emphasizes on EE in wireless communication networks, is attracting more and more attention $[11,12]$. A large amount of work has bee reported on energy-efficient design for CR networks [13-15]. For MIMO CR networks, [16] studies EE optimization problem of MIMO CR broadcast channels to improve the system throughput for unit energy consumption. In [17], the throughput and energy efficiency optimization under quality-of-service (QoS) constraints for MIMO CR systems are studied. In [18], a promising framework of spectrum sharing strategy selection based on EE is proposed for MIMO CR interference channels.

In this paper, we focus on energy-efficient power allocation for relay-aided MIMO-OFDM $\mathrm{CR}$ networks. We formulate an optimization problem related to maximization of EE of the consider network under total power constraints of cognitive source node and cognitive relay node, and interference constraints of primary users. Since the original optimization problem is difficult to solve directly, we first get an approximated problem of the original problem, and then transform the approximated problem into an equivalent convex optimization problem. A new iterative energy-efficient power allocation scheme is presented at last. The rest of this paper is organized as follows: In Section 2, we introduce the system model and formulate an energyefficient power allocation problem. In Section 3, the double-loop iterative method is presented. Finally, simulation results and Conclusions are presented in Section 4 and 5.

The following notations are used in this paper, $\mathbb{C}^{M \times N}$ denotes $M \times N$ complex matrix, $(\cdot)^{H}$ denotes the conjugate transpose, $(\cdot)^{+}$means $\max (0, \cdot)$, the distribution of a circularly symmetriccomplex-Gaussian vector with mean vector $x$ and covariance matrix $y$ is denoted by $C N(x, y)$, $\operatorname{diag}(\cdot)$ returns a square matrix with the elements of $(\cdot)$ on the diagonal.

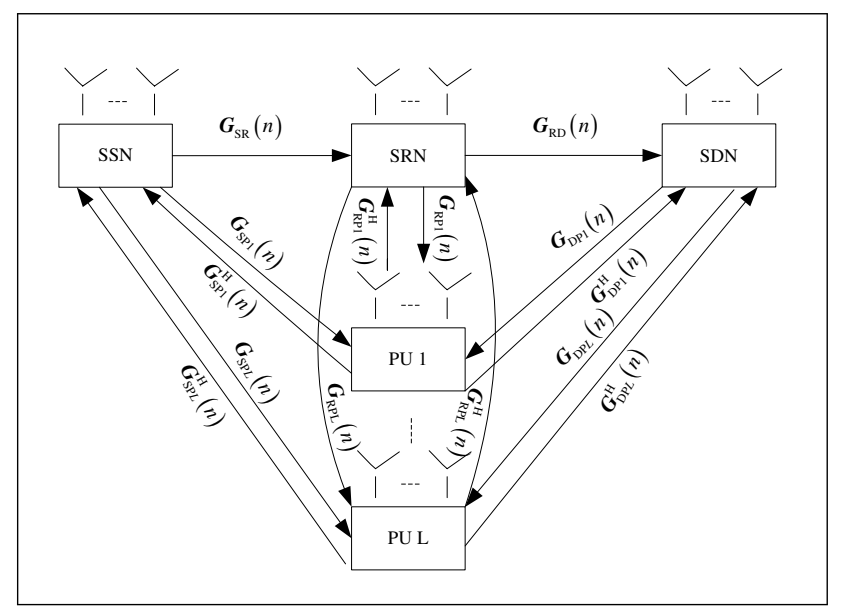

Figure 1: Relay-aided MIMO-OFDM cognitive radio network

\section{Signal Model and Problem Statement}

Consider a two-hop relay-aided cognitive radio (CR) network shown in Fig.1, there are a secondary source node (SSN), a secondary destination node (SDN), and a secondary relay node (SRN). The relay-aided CR network coexists with L licensed primary users (PUs). The SSN communicates with SDN through SRN, and they share the whole spectrum with PU. SSN, SDN, and SRN are equipped with $M_{S}$ antennas, each $\mathrm{PU}$ is equipped with $M_{P}\left(M_{P} \leq M_{S}\right.$ )antennas. The relay-aided CR network adopts OFDM modulation for transmission, and the total number of available subcarriers for CR network is $N$. Let $\boldsymbol{G}_{S R}(n) \in \mathbb{C}^{M_{S} \times M_{S}}$ and $\boldsymbol{G}_{R D}(n) \in$ $\mathbb{C}^{M_{S} \times M_{S}}$ denote the channel matrices from SSN to SRN and SRN to SDN over the $n$-th subcarrier respectively. Let $\boldsymbol{G}_{S P l}(n) \in \mathbb{C}^{M_{p} \times M_{S}}, \boldsymbol{G}_{R P l}(n) \in \mathbb{C}^{M_{p} \times M_{S}}$ and $\boldsymbol{G}_{D P l}(n) \in \mathbb{C}^{M_{p} \times M_{S}}$ denote the 
channel matrices from SSN to $l$-th PU, from SRN to $l$-PU, and from SDN to $l$-PU, respectively. The channel matrix from $l$-th PU to SSN, SDN, and SRN are $\boldsymbol{G}_{S P l}^{H}(n), \boldsymbol{G}_{R P l}^{H}(n)$ and $\boldsymbol{G}_{D P l}^{H}(n)$.

Assume there is no cooperation between CR network and PUs, environmental learning (EL) method [19] is performed to control the interference to the PUs. Via EL learning method, secondary nodes estimate the null space information of the channels between secondary nodes and PU. Assume the cognitive beamforming (CB) matrices at SSN, SRN, SDN for the n-th subcarrier are $\boldsymbol{U}_{S P l}(n) \in \mathbb{C}^{M_{S} \times\left(M_{S}-M_{P}\right)}, \boldsymbol{U}_{R P l}(n) \in \mathbb{C}^{M_{S} \times\left(M_{S}-M_{P}\right)}$ and $\boldsymbol{U}_{D P l}(n) \in \mathbb{C}^{M_{S} \times\left(M_{S}-M_{P}\right)}$, respectively. These $\mathrm{CB}$ matrices satisfy $\boldsymbol{U}_{S P l}^{H}(n) \boldsymbol{G}_{S P l}(n)=0, \boldsymbol{U}_{R P l}^{H}(n) \boldsymbol{G}_{R P l}(n)=0$ and $\boldsymbol{U}_{D P l}^{H}(n) \boldsymbol{G}_{D P l}(n)=0$. However, the accurate CB matrices are difficult to be acquired, therefore in practical applications, the estimated CB matrices $\tilde{\boldsymbol{U}}_{S P l}(n), \tilde{\boldsymbol{U}}_{R P l}(n)$ and $\tilde{\boldsymbol{U}}_{D P l}(n)$ are used.

In the first hop, the received signal at $\mathrm{CRN}$ in the $\mathrm{n}$-th subcarrier is given by:

$$
\boldsymbol{y}_{R}(n)=\boldsymbol{G}_{S R}(n) \tilde{\boldsymbol{U}}_{S P l}(n) \boldsymbol{x}_{S}+\boldsymbol{G}_{R P l}^{H}(n) \boldsymbol{w}_{P}^{1}+\boldsymbol{z}_{R}(n)
$$

where $\boldsymbol{x}_{S}$ is the transmitted signal of SSN, $\boldsymbol{w}_{P}^{1}$ is the PU interference to SRN in the first hop. $\boldsymbol{z}_{R}(n) \sim C N\left(0, \sigma_{R}^{2} \boldsymbol{I}_{M_{S}}\right)$ is the additive white Gaussian noise (AWGN) at SRN.

In the second hop, SRN first filters received signal $\boldsymbol{y}_{R}(n)$ with $\tilde{\boldsymbol{U}}_{R P l}^{H}(n)$, and then precodes the filtered signal by forwarding matrix $\boldsymbol{B}(n)$, finally precodes the resultant signal by $\tilde{\boldsymbol{U}}_{D P l}(n)$. Therefore, the received signal at $\mathrm{SDN}$ in the $n$-th subcarrier is:

$$
\boldsymbol{y}_{D}(n)=\boldsymbol{G}_{R D}(n) \tilde{\boldsymbol{U}}_{R P l}(n) \boldsymbol{B}(n) \tilde{\boldsymbol{U}}_{R P l}^{H}(n) \boldsymbol{y}_{R}(n)+\boldsymbol{G}_{D P l}^{H}(n) \boldsymbol{w}_{P}^{2}+\boldsymbol{z}_{D}(n)
$$

where $\boldsymbol{w}_{P}^{2}$ is the PU interference to SDN in the second hop, $\boldsymbol{z}_{D}(n) \sim C N\left(0, \sigma_{D}^{2} \boldsymbol{I}_{M_{S}}\right)$ is additive white Gaussian noise (AWGN) at SDN in the $n$-th subcarrier.

Finally, the CR-DN does receive CB by filtering $\boldsymbol{y}_{D}(n)$ with $\tilde{\boldsymbol{U}}_{D P l}^{H}(n)$, we have

$$
\boldsymbol{y}=\boldsymbol{D}_{2}(n) \boldsymbol{B}(n) \boldsymbol{D}_{1}(n) \boldsymbol{x}_{S}+\boldsymbol{D}(n)_{2} \boldsymbol{B}(n) \boldsymbol{n}_{1}(n)+\boldsymbol{n}_{2}(n)
$$

where $\boldsymbol{D}_{1}(n)=\tilde{\boldsymbol{U}}_{D P l}^{H}(n) \boldsymbol{G}_{R D}(n) \tilde{\boldsymbol{U}}_{R P l}(n)$ and $\boldsymbol{D}_{2}(n)=\tilde{\boldsymbol{U}}_{R P l}^{H}(n) \boldsymbol{G}_{S R}(n) \tilde{\boldsymbol{U}}_{S P l}(n), \boldsymbol{n}_{1}(n)=$ $\Delta \boldsymbol{U}_{R P l}^{H}(n) \boldsymbol{G}_{R P l}^{H}(n) \boldsymbol{w}_{P}^{1}+\tilde{\boldsymbol{U}}_{R P l}^{H}(n) \boldsymbol{z}_{R}(n), \boldsymbol{n}_{2}(n)=\Delta \boldsymbol{U}_{D P l}^{H}(n) \boldsymbol{G}_{D P l}^{H}(n) \boldsymbol{w}_{P}^{2}+\tilde{\boldsymbol{U}}_{D P l}^{H}(n) \boldsymbol{z}_{D}(n)$, $\Delta \boldsymbol{U}_{R P l}^{H}(n)=\tilde{\boldsymbol{U}}_{R P l}^{H}(n)-\boldsymbol{U}_{R P l}^{H}(n), \Delta \boldsymbol{U}_{D P l}^{H}(n)=\tilde{\boldsymbol{U}}_{D P l}^{H}(n)-\boldsymbol{U}_{D P l}^{H}(n)$ denote the first-order perturbations of the CB matrices due to imperfect environmental learning [8, 19].

Let the singular value decomposition of $\left\{\boldsymbol{D}_{k}(n)\right\}_{k=1,2}$ be $\boldsymbol{D}_{k}(n)=U_{k}(n) \boldsymbol{\Lambda}_{k}(n) \boldsymbol{V}_{k}^{H}(n)$ $(k=1,2)$, and define $q_{m, n}=\sqrt{\frac{p_{m, n}^{R}}{p_{m, n}^{S} \lambda_{m, n}^{S R}+\sigma_{R}^{2}}}$, where $p_{m, n}^{S}$ and $p_{m, n}^{R}$ are the transmit power of SSN and SRN, $\lambda_{m, n}^{S R}$ is the eigenvalue of $\boldsymbol{G}_{S R}(n)$. Thus, the forwarding matrix can be defined as $\boldsymbol{B}(n)=\boldsymbol{V}_{2}^{H}(n) \boldsymbol{Q}(n) \boldsymbol{U}_{1}(n)$. Multiplying $y$ with $U_{2, n}^{H}$ at SDN, we have

$$
\boldsymbol{y}=\boldsymbol{\Lambda}_{2}(n) \boldsymbol{\Sigma}(n) \boldsymbol{\Lambda}_{1}(n) \boldsymbol{V}_{1}(n) \boldsymbol{x}_{S}+\boldsymbol{\Lambda}_{2}(n) \boldsymbol{\Sigma}(n) \boldsymbol{U}_{1}^{H}(n) \boldsymbol{n}_{1}(n)+\boldsymbol{U}_{2}^{H}(n) \boldsymbol{n}_{2}(n)
$$

where $\boldsymbol{\Sigma}_{n}=\operatorname{diag}\left(q_{1, n}, q_{2, n}, \cdots, q_{M, n}\right)$.

According to formula (4), the MIMO-OFDM channel between SSN and SDN can be decomposed into $N \times M_{S}$ parallel independent channels, therefore, the throughput of the Relay-aided MIMO-OFDM network is

$$
C_{t p}(\mathrm{p})=\frac{1}{2} \sum_{m=1}^{M} \sum_{n=1}^{N} \log _{2}\left(1+\frac{p_{m, n}^{S} \alpha_{m, n} p_{m, n}^{R} \beta_{m, n}}{1+p_{m, n}^{S} \alpha_{m, n}+p_{m, n}^{R} \beta_{m, n}}\right)
$$

where $\alpha_{m, n}=\frac{\lambda_{m, n}^{S R}}{\sigma_{R}^{2}+\psi_{1}}, \beta_{m, n}=\frac{\lambda_{m, n}^{R D}}{\sigma_{D}^{2}+\psi_{2}}, \lambda_{m, n}^{R D}$ is the eigenvalue of $\boldsymbol{G}_{R D}(n), \psi_{1}$ and $\psi_{2}$ are constants and linear with $\frac{1}{N_{E L}}\left(N_{E L}\right.$ is the number of samples in EL stage), $\mathrm{p}=\left\{p_{m, n}^{S}, p_{m, n}^{R}\right\}$ is power 
vector. The overall power consumption at SSN and SDN can be expressed respectively as follows:

$$
\begin{aligned}
P^{S S N} & =\tau^{S} \sum_{m=1}^{M} \sum_{n=1}^{N} p_{m, n}^{S}+P_{c}^{S} \\
P^{S R N} & =\tau^{R} \sum_{m=1}^{M} \sum_{n=1}^{N} p_{m, n}^{R}+P_{c}^{R} .
\end{aligned}
$$

where $\tau^{S}$ and $P_{c}^{S}$ are the reciprocal of drain efficiency of power amplifier and circuit power at SSN. $\tau^{R}$ and $P_{c}^{R}$ are the reciprocal of drain efficiency of power amplifier and circuit power at SDN.

The EE of the cognitive relay network while selecting the $l$-th CRN for transmitting is defied as:

$$
\xi^{E E}(\mathrm{p})=\frac{\frac{1}{2} \sum_{m=1}^{M} \sum_{n=1}^{N} \log _{2}\left(1+\frac{p_{m, n}^{S} \alpha_{m, n} p_{m, n}^{R} \beta_{m, n}}{1+p_{m, n}^{S} \alpha_{m, n}+p_{m, n}^{R} \beta_{m, n}}\right)}{\tau^{S} \sum_{m=1}^{M} \sum_{n=1}^{N} p_{m, n}^{S}+\tau^{R} \sum_{m=1}^{M} \sum_{n=1}^{N} p_{m, n}^{R}+P_{c}^{S}+P_{c}^{R}}
$$

Since we use the estimated CB matrices, the interferences to PUs cased by SSN and SRN are inevitably, the interferences cased by SSN and SRN to $l$-th PU are

$$
\begin{gathered}
I_{l}^{S P}=\frac{\mu_{S}}{\sigma_{l}^{2}} \sum_{m=1}^{M} \sum_{n=1}^{N} p_{m, n}^{S} \\
I_{l}^{R P}=\frac{\mu_{R}}{\sigma_{l}^{2}} \sum_{m=1}^{M} \sum_{n=1}^{N} p_{m, n}^{R} .
\end{gathered}
$$

where $\mu_{S}$ and $\mu_{R}$ are constants and linear with $\frac{1}{N_{E L}}, \sigma_{l}^{2}$ is the transmit power of the $l$-th PU signal.

From (7), the objective of energy-efficient power allocation problem for the relay-aided MIMO-OFDM CR network can be expressed as:

$$
\mathrm{OP} 1 \max _{p_{m, n}^{S}, p_{m, n}^{R} \geq 0} \frac{\frac{1}{2} \sum_{m=1}^{M} \sum_{n=1}^{N} \log _{2}\left(1+\frac{p_{m, n}^{S} \alpha_{m, n} p_{m, n}^{R} \beta_{m, n}}{1+p_{m, n}^{S} \alpha_{m, n}+p_{m, n}^{R} \beta_{m, n}}\right)}{\tau^{S} \sum_{m=1}^{M} \sum_{n=1}^{N} p_{m, n}^{S}+\tau^{R} \sum_{m=1}^{M} \sum_{n=1}^{N} p_{m, n}^{R}+P_{c}^{S}+P_{c}^{R}}
$$

subject to

$$
\left\{\begin{array}{l}
C 1: \sum_{m=1}^{M} \sum_{n=1}^{N} p_{m, n}^{S} \leq P_{t h}^{S} \\
C 2: \sum_{m=1}^{M} \sum_{n=1}^{N} p_{m, n}^{R} \leq P_{t h}^{R} \\
C 3: \frac{\mu_{S}}{\sigma_{l}^{2}} \sum_{m=1}^{M} \sum_{n=1}^{N} p_{m, n}^{S} \leq I_{t h}, l=1,2, \cdots, L \\
C 4: \frac{\mu_{R}}{\sigma_{l}^{2}} \sum_{m=1}^{M} \sum_{n=1}^{N} p_{m, n}^{R} \leq I_{t h}, l=1,2, \cdots, L .
\end{array}\right.
$$

where $P_{t h}^{S}$ and $P_{t h}^{R}$ are the total power budgets of SSN and SRN respectively, $I_{t h}$ is the interference threshold of PUs. C1 and C2 are transmission power constraints of SSN and SDN, $\mathrm{C} 3$ and $\mathrm{C} 4$ are the interference constraints of the PUs.

Duo to lack of convexity, it is difficult to solving OP1 directly. In the following, we make a joint concave approximation to OP1 and introduce a new equivalent optimization problem via nonlinear fractional programming (NFP) [21]. 


\section{Energy Efficient Power Allocation Algorithm}

To make OP1 more tractable, the throughput $C_{t p}$ can be approximated at the high signalto-noise ratio (SNR) as

$$
\tilde{C}_{t p}(\mathrm{p})=\frac{1}{2} \sum_{m=1}^{M} \sum_{n=1}^{N} \log _{2}\left(1+\frac{p_{m, n}^{S} \alpha_{m, n} p_{m, n}^{R} \beta_{m, n}}{p_{m, n}^{S} \alpha_{m, n}+p_{m, n}^{R} \beta_{m, n}}\right)
$$

Note: As in [20], $\tilde{C}_{t p}(\mathrm{p})$ is joint concave with $p_{m, n}^{S}$ and $p_{m, n}^{R}$.

Thus, we can also get the approximation of EE as:

$$
\tilde{\xi}^{E E}(\mathrm{p})=\frac{\tilde{C}_{t p}(\mathrm{p})}{P_{\text {total }}(\mathrm{p})}
$$

where $P_{\text {total }}(\mathrm{p})=P^{S S N}+P^{S R N}$.

Substitute $\tilde{\xi}^{E E}$ into OP1, we get the approximated optimization problem

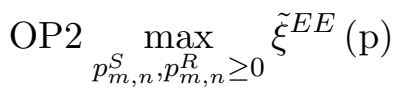

subject to

$$
C 1 \sim C 4
$$

For notational simplicity, we define $\aleph$ as the set of feasible solution of OP2, and let $\mathrm{p}=$ $\left\{p_{m, n}^{S}, p_{m, n}^{R}\right\}$ be variable vector. Define the maximum EE $\rho^{*}$ of network as follows:

$$
\rho^{*}=\frac{\tilde{C}_{t p}\left(\mathrm{p}^{*}\right)}{P_{\text {total }}\left(\mathrm{p}^{*}\right)}=\max _{p \in \aleph} \frac{\tilde{C}_{t p}(\mathrm{p})}{P_{\text {total }}(\mathrm{p})}
$$

where $\mathrm{p}^{*}$ is the optimal solution of OP2.

Introducing a new parametric optimization problem OP3

$$
\text { OP3 } \max _{p_{m, n}^{S}, p_{m, n}^{R} \geq 0}\left\{\tilde{C}_{t p}(\mathrm{p})-\rho P_{\text {total }}(\mathrm{p})\right\}
$$

subject to

$$
C 1 \sim C 4
$$

where $\rho$ is non-negative parameter. Since $\tilde{C}_{t p}(p)$ is joint concave with $p_{m, n}^{S}$ and $p_{m, n}^{R}$, for a given $\rho, \mathrm{OP} 3$ is a convex optimization problem.

Next, introduce a theorem based on NFP [21]: Theorem The optimal solution achieve the maximum EE if and only if

$$
\begin{aligned}
& \max _{p \in \aleph}\left\{\tilde{C}_{t p}(\mathrm{p})-\rho^{*} P_{\text {total }}(\mathrm{p})\right\} \\
= & \tilde{C}_{t p}\left(\mathrm{p}^{*}\right)-\rho^{*} P_{\text {total }}\left(\mathrm{p}^{*}\right) \\
= & 0
\end{aligned}
$$

with $\tilde{C}_{t p}(\mathrm{p}) \geq 0, P_{\text {total }}(\mathrm{p})>0$.

Proof: Similar proof can be found in [21].

The Theorem implies that for fractional OP2, there is an equivalent problem whose objective function is in subtractive form, e.g. $\tilde{C}_{t p}(\mathrm{p})-\rho^{*} P_{\text {total }}(\mathrm{p})$. Therefore, solving OP2 is equivalent to solve problem OP3 for a given $\rho$ and then update $\rho$ until the Theorem is satisfied. 
An alternative method solving OP3 is through deriving the Lagrange dual [22] of the optimization problem OP3. The Lagrange function of OP3 is defined as bellow:

$$
\begin{aligned}
& L_{a g}\left(p, \theta_{1}, \theta_{2},\left\{\chi_{l}\right\}_{l=1}^{L},\left\{\eta_{l}\right\}_{l=1}^{L}\right)=\frac{1}{2} \sum_{m=1}^{M} \sum_{n=1}^{N} \log _{2}\left(1+\frac{p_{m, n}^{S} \alpha_{m, n} p_{m, n}^{R} \beta_{m, n}}{p_{m, n}^{S} \alpha_{m, n}+p_{m, n}^{R} \beta_{m, n}}\right) \\
& -\rho\left[\tau^{S} \sum_{m=1}^{M} \sum_{n=1}^{N} p_{m, n}^{S}+\tau^{R} \sum_{m=1}^{M} \sum_{n=1}^{N} p_{m, n}^{R}+P_{C}^{S}+P_{C}^{R}\right] \\
& -\theta_{1}\left(\sum_{m=1}^{M} \sum_{n=1}^{N} p_{m, n}^{S}-P_{t h}^{S}\right)-\theta_{2}\left(\sum_{m=1}^{M} \sum_{n=1}^{N} p_{m, n}^{R}-P_{t h}^{R}\right) \\
& -\sum_{l=1}^{L} \chi_{l}\left(\frac{\mu_{S}}{\sigma_{l}^{2}} \sum_{m=1}^{M} \sum_{n=1}^{N} p_{m, n}^{S}-I_{t h}\right)-\sum_{l=1}^{L} \eta_{l}\left(\frac{\mu_{R}}{\sigma_{l}^{2}} \sum_{m=1}^{M_{S}} \sum_{n=1}^{N} p_{m, n}^{R}-I_{t h}\right)
\end{aligned}
$$

where $\theta_{1}, \theta_{2}, \chi_{l}$ and $\eta_{l}$ are the Lagrange multipliers.

Therefore, the Lagrange dual function of the primal problem OP3 can be written as:

$$
D_{u a l}\left(\theta_{1}, \theta_{2},\left\{\chi_{l}\right\}_{l=1}^{L},\left\{\eta_{l}\right\}_{l=1}^{L}\right)=\max _{p \geq 0} L_{a g}\left(p, \theta_{1}, \theta_{2},\left\{\chi_{l}\right\}_{l=1}^{L},\left\{\eta_{l}\right\}_{l=1}^{L}\right)
$$

The corresponding Lagrangian dual problem of OP3 can be expressed as:

$$
\min _{\theta_{1}, \theta_{2}, \chi_{l}, \eta_{l} \geq 0} D_{\text {ual }}\left(\theta_{1}, \theta_{2},\left\{\chi_{l}\right\}_{l=1}^{L},\left\{\eta_{l}\right\}_{l=1}^{L}\right)
$$

The problem (17) is convex, according to the Karush-Kuhn-Tucker condition: $\frac{\partial L_{a g}}{\partial p_{m, n}^{S}}=0$ and $\frac{\partial L_{a g}}{\partial p_{m, n}^{R}}=0$, then we have:

$$
\begin{aligned}
& \frac{1}{2 \ln 2} \frac{\alpha_{m, n} \beta_{m, n}^{2}\left(p_{m, n}^{R}\right)^{2}}{\left(p_{m, n}^{S} \alpha_{m, n}+p_{m, n}^{R} \beta_{m, n}\right)\left(p_{m, n}^{S} \alpha_{m, n}+p_{m, n}^{R} \beta_{m, n}+p_{m, n}^{S} p_{m, n}^{R}\right)}=\rho \tau^{S}+\theta_{1}+\mu_{S} \sum_{l=1}^{L} \frac{\chi_{l}}{\sigma_{l}^{2}} \\
& \frac{1}{2 \ln 2} \frac{\alpha_{m, n}^{2} \beta_{m, n}\left(p_{m, n}^{S}\right)^{2}}{\left(p_{m, n}^{S} \alpha_{m, n}+p_{m, n}^{R} \beta_{m, n}\right)\left(p_{m, n}^{S} \alpha_{m, n}+p_{m, n}^{R} \beta_{m, n}+p_{m, n}^{S} p_{m, n}^{R}\right)}=\rho \tau^{R}+\theta_{2}+\mu_{R} \sum_{l=1}^{L} \frac{\eta_{l}}{\sigma_{l}^{2}}
\end{aligned}
$$

Solving the above two equations, we get the optimal power allocation solutions as:

$$
\begin{aligned}
& p_{m, n}^{S}=\frac{1}{\sqrt{\alpha_{m, n} x_{1}}\left(\sqrt{\frac{x_{1}}{\alpha_{m, n}}}+\sqrt{\frac{x_{2}}{\beta_{m, n}}}\right)}\left(1-\left(\sqrt{\frac{x_{1}}{\alpha_{m, n}}}+\sqrt{\frac{x_{2}}{\beta_{m, n}}}\right)^{2}\right)^{+} \\
& p_{m, n}^{R}=\frac{1}{\sqrt{\beta_{m, n} x_{2}}\left(\sqrt{\frac{x_{1}}{\alpha_{m, n}}}+\sqrt{\frac{x_{2}}{\beta_{m, n}}}\right)}\left(1-\left(\sqrt{\frac{x_{1}}{\alpha_{m, n}}}+\sqrt{\frac{x_{2}}{\beta_{m, n}}}\right)^{2}\right)^{+}
\end{aligned}
$$

where $[x]^{+}=\max (0, x), x_{1}=\rho \tau^{S}+\theta_{1}+\mu_{S} \sum_{l=1}^{L} \frac{\chi_{l}}{\sigma_{l}^{2}}$ and $x_{2}=\rho \tau^{R}+\theta_{2}+\mu_{R} \sum_{l=1}^{L} \frac{\eta_{l}}{\sigma_{l}^{2}}$. Note: (21) and (22) show that $p_{m, n}^{S}$ and $p_{m, n}^{R}$ are either both positive or both zero, this implies that if power allocated to the $n$-th subcarrier in the first hop is zero, then no power is allocated to its corresponding subcarrier in the second hop, which meets the intuition very well. 
Table 1:

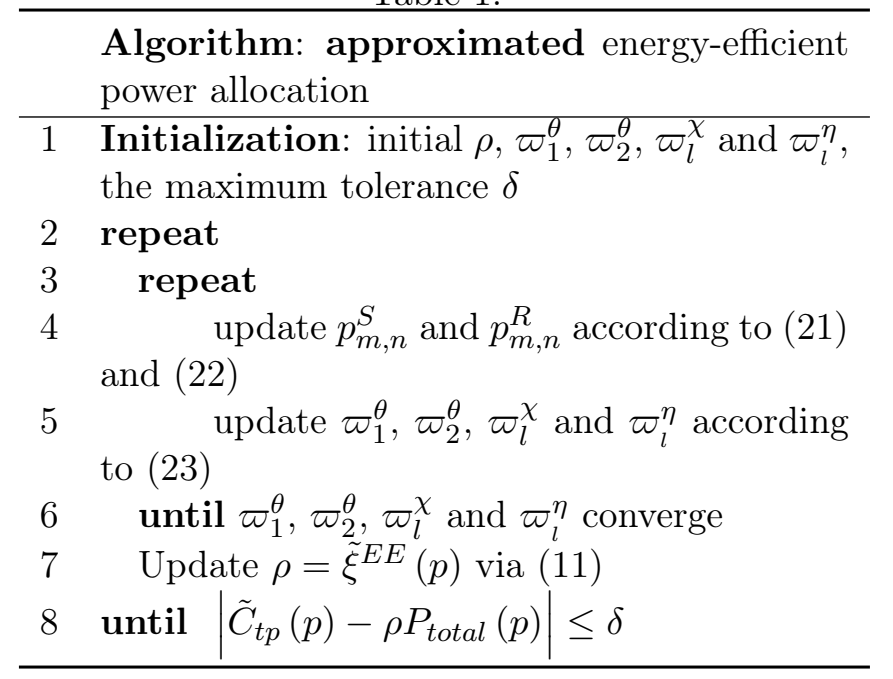

The optimal dual variables can be obtained from the dual problem (18) using the subgradient method[23]. The dual variables could be updated as:

$$
\begin{aligned}
& \theta_{1}=\left(\theta_{1}+\varpi_{1}^{\theta}\left(P_{t h}^{S}-\sum_{m=1}^{M} \sum_{n=1}^{N} p_{m, n}^{S}\right)\right)^{+} \\
& \theta_{2}=\left(\theta_{2}-\varpi_{2}^{\theta}\left(P_{t h}^{R}-\sum_{m=1}^{M} \sum_{n=1}^{N} p_{m, n}^{R}\right)\right)^{+} \\
& \chi_{l}=\left(\chi_{l}-\varpi_{l}^{\chi}\left(I_{t h}-\frac{\mu_{S}}{\sigma_{l}^{2}} \sum_{m=1}^{M} \sum_{n=1}^{N} p_{m, n}^{S}\right)\right)^{+} \\
& \eta_{l}=\left(\eta_{l}-\varpi_{l}^{\eta}\left(I_{t h}-\frac{\mu_{R}}{\sigma_{l}^{2}} \sum_{m=1}^{M} \sum_{n=1}^{N} p_{m, n}^{R}\right)\right)^{+}
\end{aligned}
$$

where $\varpi_{1}^{\theta}, \varpi_{2}^{\theta}, \varpi_{l}^{\chi}$ and $\varpi_{l}^{\eta}$ are the step length. According to the aforementioned analysis, we propose a two loop iterative algorithm to solve the approximated energy-efficient power allocation problem OP2, which is termed as AEE-PA and tabulated as in Table 1.

Note: [23] shows that the subgradient algorithm can converge to the optimal solution of convex optimization problems within a small range. Therefore, the inner loop can converge to the optimal solution of the dual problem (18) with in a small range. Since OP3 is convex optimization problem, the duality gap for OP3 is zero, the inner loop also converges to the optimal solution of OP3 within a small range. The detailed proves of the convergence of the outer loop, i.e. NFP can be found in [21].

\section{Performance Simulations}

We perform numerical simulations to evaluate the present some numerical experiments to evaluate the performance of our proposed scheme. Without loss of generality, the channel gains are assumed to be Rayleigh fading with an average power gain of $1 \mathrm{~dB}$, and set the parameters $N=10, L=2, M_{S}=4, M_{P}=2, \sigma_{R}^{2}=\sigma_{D}^{2}=10^{-6} W, \sigma_{l}^{2}=1 W, \tau^{S}=\tau^{R}=1$, $P_{c}^{S}=P_{c}^{R}=10^{-2} W, P_{t h}^{S}=P_{t h}^{R}=P_{\max }$. Since $\psi_{1}, \psi_{2}, \mu_{S}$ and $\mu_{R}$ are linear with $\frac{1}{N_{E L}}$, for 
simplicity, let, , and are equals with. All the results have been averaged over 500 iterations. We compare the proposed algorithm with the traditional throughput maximum problem. Change the objective function $\xi^{E E}(\mathrm{p})$ in OP1 with the throughput $C_{t p}(\mathrm{p})$ in (5) and change the approximated objective function $\tilde{\xi}^{E E}(\mathrm{p})$ in OP2 with the approximated throughput $\tilde{C}_{t p}(\mathrm{p})$ in (10), then we formulate the traditional throughput maximum problem. Since $\tilde{C}_{t p}(\mathrm{p})$ is joint concave in with $p_{m, n}^{S}$ and $p_{m, n}^{R}$, the throughput maximum problem is convex problem which can be solved by many standard convex optimization algorithms [22]. We name the method to solve the throughput maximum problem as TM-PA. In the following, we compare the proposed algorithm with the TM-PA scheme.

Since the proposed AEE-PA consists of two loops, we only consider the affect of the number of outer loop iterations $t_{O}$ and set the number of inner iterations large enough to guarantee that the inner loop can find the optimal solution of OP3. Fig.2 shows the EE versus the outer loop iterations $t_{O}$ for different total power budget under $I_{t h}=1 \mathrm{~W}, N_{E L}=500$. It can be observed in Fig.2 that AEE-PA converges to the optimal value within eleven iterations for all considered value of total power budgets. The maximum EE can be improved when there are more total power budgets.

Fig.3 depicts the EE versus total power budget $P_{\max }$ for different interference thresholds under $N_{E L}=500$. As shown in Fig.3, the EE of the both algorithms increases with the increasing of the total power budget, however the proposed AEE-PA has a higher EE than the non energy efficiency scheme TM-PA. The EE versus interference threshold $I_{t h}$ for different total power budgets under $N_{E L}=500$ is evaluated in Fig.4. It is shown in Fig.4 that the EE of the both algorithms grows with the growth of the interference threshold. This is because that the lower the interference threshold is, the more the CR network suffers outage.

We also evaluate the impact of $N_{E L}$ (the number of samples in EL stage) on the proposed algorithm. In Fig.5, EE versus interference threshold $I_{t h}$ for different $N_{E L}$ under $P_{\max }=0.5 \mathrm{~W}$ is depicted. Obviously, the algorithm has a better performance with lager $N_{E L}$ than small $N_{E L}$. This is because smaller $N_{E L}$ performs poor learning and yields large interference to PUs.

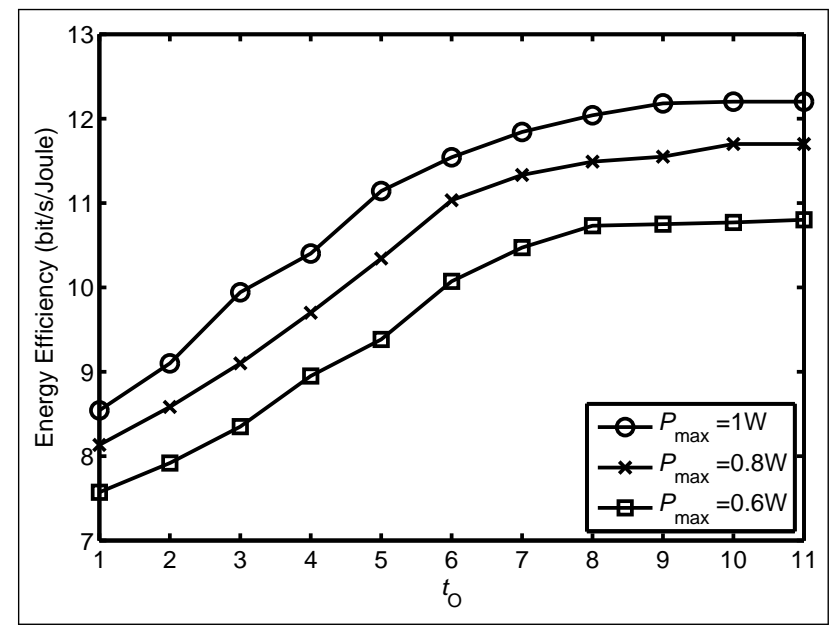

Figure 2: Energy efficiency versus the outer iterations for different total power budget 


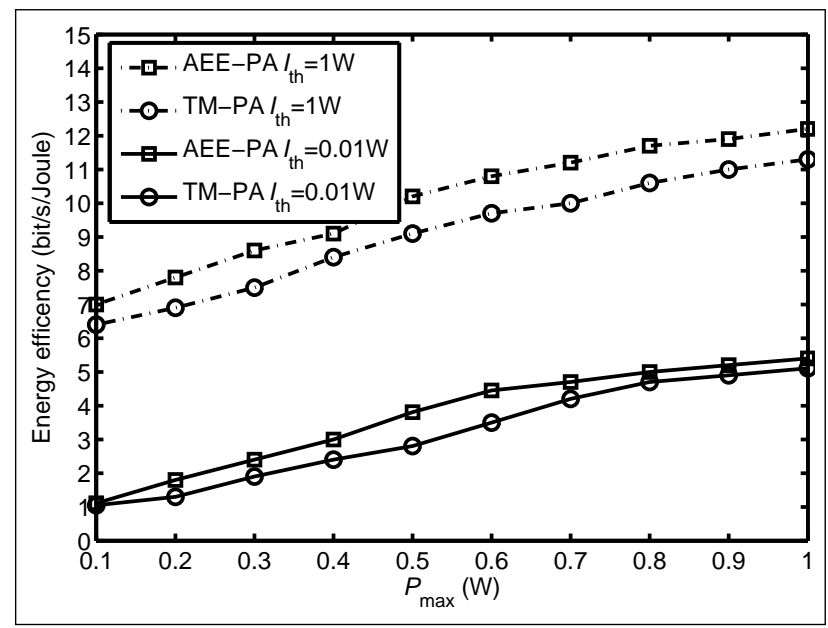

Figure 3: Energy-efficiency versus total power budget for different interference threshold

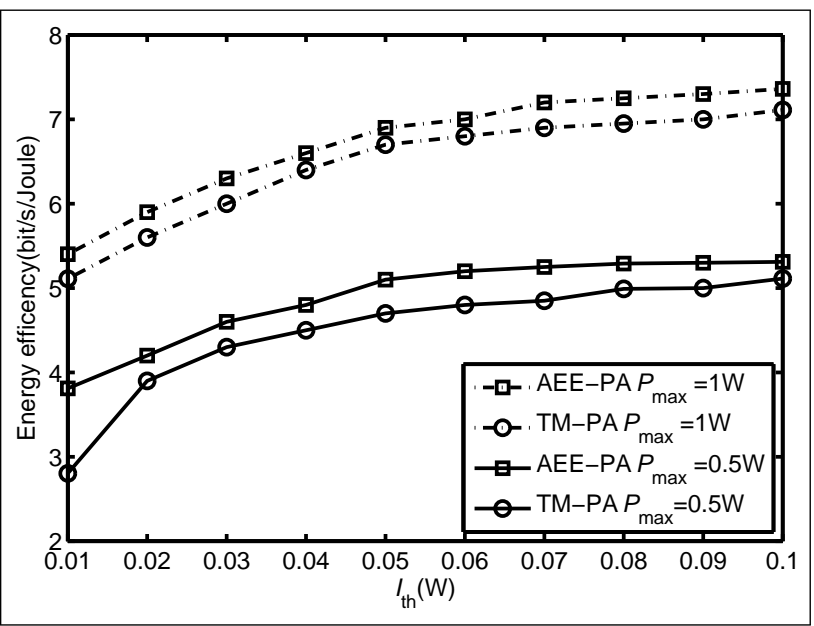

Figure 4: Energy efficiency versus interference threshold for different total power budget

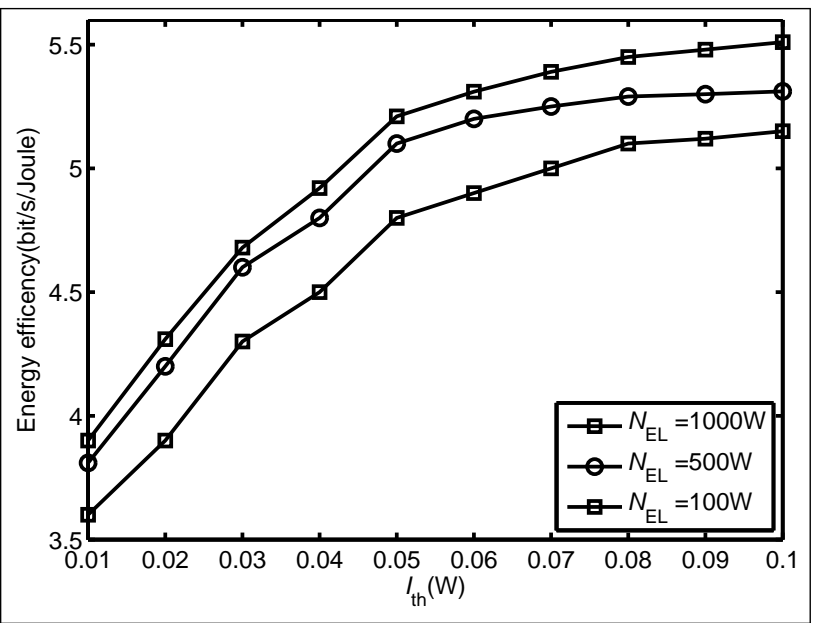

Figure 5: Energy efficiency versus interference threshold for different $N_{E L}$ 


\section{Conclusions}

In this paper, we investigated the power allocation for relay-aided MIMO-OFDM cognitive radio networks from energy efficiency perspective. Different from traditional throughput maximizing methods, we solve the power allocation problem via maximizing the energy efficiency measured by "Joule per bit" metric. However, the formulated problem is nonconvex. To make it solvable, we first make an approximation to the original problem. Indeed, the approximated problem is a fractional programming problem. Then, the approximated problem is transformed into a parametric convex optimization problem. Finally, we give closed form solutions to the parametric convex optimization problem and proposed a two loop iterative energy-efficient power allocation algorithm. To show the improvement in energy efficiency, we compared the proposed algorithm with the traditional throughput maximizing method. From the simulation results, we observed that the proposed new scheme have a better performance than conventional capacity maximization scheme in energy efficiency.

\section{Bibliography}

[1] L. Lu, G.Y. Li, A. Maaref, et. al. (2014), Opportunistic transmission exploiting frequency- and spatial-domain degrees of freedom, IEEE Wireless Communications, April 2014, 21(2):91-97.

[2] Muhammad Naeem, Alagan Anpalagan, Muhammad Jaseemuddin, et. al. (2014), Resource allocation techniques in cooperative cognitive radio networks, IEEE Communications Surveys 86 Tutorials, Second Quarter 2014, 16(2):729-744.

[3] Mehdi Ghamari Adian and Hassan Aghaeinia (2014), Optimal and sub-optimal resource allocation in multiple-input multiple-output-orthogonal frequency division multiplexingbased multi-relay cooperative cognitive radio networks, IET Communications, March 2014, 8(5):646-657.

[4] Quanzhong Li, Qi Zhang, Renhai Feng, et. al. (2013), Optimal relay selection and beamforming in MIMO cognitive multi-relay networks, IEEE Communications Letters, June 2013, 17(6):1188-1191.

[5] Sha Hua, Hang Liu, Xuejun Zhuo, et.al. (2014), Exploiting multiple antennas in cooperative cognitive radio networks, IEEE Transactions on Vehicular Technology, February 2014, 99: $1-12$.

[6] Ahmad Alsharoa, Hakinm Ghazzai and Mohamed-Slim Alouini (2014), Optimal transmit power allocation for MIMO two-way cognitive relay networks with multiple relays using AF strategy, IEEE Wireless Communications Letters, February 2014, 3(1):30-33.

[7] Mehdi Ghamari Adian and Hassan Aghaeinia (2014), Resoure allocation in MIMO-OFDM based cooperative cognitive radio networks, IEEE Transactions on Communications, May 2014, 99: 1-11.

[8] Shou Li, Bingquan Li, Chengwen Xing, et. al.(2013), Joint resource allocation for learningbased cognitive radio networks with MIMO-OFDM relay-aided transmissions, 2013 IEEE Wireless Communications and Networking Conference, 7-10 April 2013, Shanghai, 327132676 .

[9] G. P. Fettweis and E. Zimmermann (2008), ICT energy consumption-trends and challenges, Proceedings in 11-th International Symposiums Wireless Personal Multimedia Communications, Lapland, Finland, September 2008, 1-4. 
[10] Chunlong He, Geoffrey Ye Li, Fu-Chun Zheng, et.al (2014), Energy-efficient resource allocation in OFDM Systems with distributed antennas, IEEE Transactions on Vehicular Technolgoy, March 2014, 63(3):1223-1231.

[11] Geoffrey Ye Li, Zhikun Xu, Cong Xiong, et.al.(2011), Energy-efficient wireless communications: tutorial, survey, and open issues, IEEE Wireless Communications, December 2011, 18(6):28-35.

[12] Cong Xiong, Lu Lu and Geoffery Ye Li (2014), Energy-efficient specturm access in cognitive radios, IEEE Journal of Selected Areas in Communicatinos, March 2014, 32(3):550-562.

[13] Cong Xiong, Geoffery Ye Li, Yalin Liu, et.al. Energy-efficient design for downlink OFDMA with delay-sensitive traffic, IEEE Transactions on Wireless Communications, June 2013, 12(6):3085-3095.

[14] Suzan Bayhan and Fatih Alagoz (2013), Schedulign in centralized cognitive radio networks for energy efficiency, IEEE Transactions on Vehicular Technolgoy, February 2013, 62(2):582595.

[15] Seonwook Kim, Byeong Gi Lee (2014), Energy-per-bit minimized radio resource allocation in heterogeneous networks, IEEE Transactions on Wireless Communications, April 2014, 13(4):1862-1873.

[16] Junling Mao, Gang Xie, Jinchun Gao, et al.(2013), Energy efficiency optimization for cognitive radio MIMO broadcast channels, IEEE Communications Letters, February 2013, 17(2):337-340.

[17] Sami Akin and Mustafa Cenk Gursoy (2013), On the throughput and energy efficiency of cognitive MIMO transmissions, IEEE Transactions on Vehicular Technology, March 2013, 62(7):3245-3260.

[18] Wei Zhong, and Jiaheng Wang (2013), Energy efficient spectrum sharing strategy selection for cognitive MIMO interference channels, IEEE Transactions Signal Processing, July 2013, 61(14):3705-3717.

[19] Feifei Gao, Rui Zhang, Ying-Chang Liang, et.al.(2010), Design of learning-based MIMO cognitive radio systems, IEEE Transactions on Vehicular Technology, May 2010, 59(4):17071720 .

[20] Ingmar Hammerstrom and Armin Wittneben (2007), Power allocation schemes for amplifyand-forward MIMO-OFDM relay links, IEEE Transactions on Wireless Communications, August 2007, 6(8):2798-2802.

[21] Werner Dinkelbach (1967), On Nonlinear Fractional Programming, Management Science, March 1967, 13(7):492-498.

[22] Stephen Boyd and Lieven Vandenberghe (2014), Convex Optimization, Cambridge University Press, 2004.

[23] Stephen Boyd, L. Xiao and A. Mutapcic, Subgradient Methods, Online: https://web.stanford.edu/class/ee392o/subgrad_method.pdf. 